\title{
Les mots et les choses : Johannes Schefferus et la philologie du réel entre Rhin et Laponie
}

\section{Thomas Mohnike}

\section{(2) OpenEdition}

1 Journals

Édition électronique

URL : https://journals.openedition.org/rbnu/1942

DOI : 10.4000/rbnu. 1942

ISSN : 2679-6104

Éditeur

Bibliothèque nationale et universitaire de Strasbourg

Édition imprimée

Date de publication : 1 novembre 2013

Pagination : 8-17

ISBN : 9782859230517

ISSN : 2109-2761

Référence électronique

Thomas Mohnike, «Les mots et les choses : Johannes Schefferus et la philologie du réel entre Rhin et Laponie », La Revue de la BNU [En ligne], 8 | 2013, mis en ligne le 01 novembre 2013, consulté le 17 mai 2021. URL : http://journals.openedition.org/rbnu/1942 ; DOI : https://doi.org/10.4000/rbnu.1942

\section{(c) (i) (8)}

La Revue de la BNU est mise à disposition selon les termes de la Licence Creative Commons Attribution - Pas d'Utilisation Commerciale - Partage dans les Mêmes Conditions 4.0 International. 
cenzanulscitit a este compose

entangue Surotise par Olaus Grane prosteur futhevien delapvouince de Pitha pour Pinstruction des fapors qui esfoient Sonesaconguite. if auoit esté auparauantmusicive de laveise Christine. Oesuede, je serrestoit ausside fastronzonzic ct de fástrologie indiciaire, dela chimue er de la rzedecine, if estoit gendre dre - farrex founnes Jovreks pasteur de la proucinede Jovra dorrlespremoinessurla laporie ont beaceony serui a 77. Scheffir poucr composer Phistoire quikerza faite.

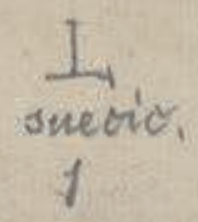




\section{Les mots et les choses : \\ Johannes Schefferus et la philologie du réel entre Rhin et Laponie}

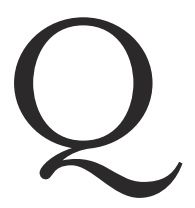

uand j'ai appris pour la première fois l'existence d'un catalogue électronique des manuscrits conservés en France, j'ai tout de suite regardé si Strasbourg disposait de manuscrits d'origine nordique. Je ne m'attendais pas à une grande découverte, mais l'un des manuscrits trouvés m'a profondément intrigué par l'improbabilité de son existence strasbourgeoise : il s'agit d'un rapport du $17^{\mathrm{e}}$ siècle écrit par un pasteur suédois, Olaus Graan, qui exerçait son office tout au nord du pays, à Piteå. Son texte traite des mœurs et des coutumes des Sames, peuple habitant la région communément appelée la Laponie. Comment un rapport d'une telle " nordicité », pour ainsi dire, avait-il pu trouver le chemin de Strasbourg?

La découverte de ce manuscrit fut pour moi l'occasion d'un profond étonnement, d'une expérience d'étrangeté dans mes attentes quotidiennes qui m'a conduit à me questionner. C'était pour ainsi dire un exemple du phénomène que le célèbre théoricien français Roland Barthes a jadis appelé l'expérience du "punctum " : le vécu d'une singularité qui crée un " effet de ponctuation ", un arrêt de pensée qui mène à la réflexion et parfois à la découverte de quelque chose de nouveau. Un rapport du $17^{\mathrm{e}}$ siècle, rédigé aux alentours du cercle polaire par un prêtre suédois, qui se retrouve dans une bibliothèque de Strasbourg ! C'était pour moi quelque chose de presque plus étrange et exotique que la présence de la fameuse momie égyptienne dans la collection de cette dernière.

Quelques questions suivirent cette découverte : qui était ce pasteur suédois, pourquoi et dans quel but avaitil rédigé son rapport ? À qui avait-il envoyé ce document, et pour quelle raison l'avait-on proposé à la collection strasbourgeoise ? Et pourquoi avait-on décidé de le garder, de le conserver ? Quel intérêt pour le Grand Nord se cachait derrière cet écrit ? Les réponses à ces interrogations font apparaître, de façon tout à fait passionnante, un paysage des pratiques du savoir et de sa circulation entre la région rhénane et la Laponie à la fin du $17^{\mathrm{e}}$ et au cours du $18^{\mathrm{e}}$ siècle.

Hélas, nos sources ne nous disent pas grand-chose sur les circonstances exactes de l'acquisition du manuscrit ${ }^{1}$. Sur la première page, nous pouvons lire en français (nous retranscrivons le texte littéralement):

"Ce manuscrit a esté composé en langue suédoise par Olaus Graan pasteur luthérien de la province de Pitha pour l'instruction des Lapons qui estoient souz sa conduite. Il avoit esté auparavant musicien de la reine Christine de Suède, il se mesloit aussi de l'astronomie et de l'astrologie judiciaire, de la chimie et de la médecine, il estoit gendre du fameux Joannes Torneus pasteur de la province de Torna dont les mémoires sur la Laponie ont beaucoup servi à M. Scheffer pour composer l'histoire qu'il en a faite ».

Malheureusement, l'auteur de cette notice nous est inconnu. Elle semble avoir été rédigée quelque temps après la rédaction du rapport. La langue employée pourrait indiquer que son auteur vivait au $18^{\mathrm{e}}$ siècle ; il était peutêtre strasbourgeois, mais utilisait la langue française et venait donc, en tout cas, d'une couche sociale plutôt privilégiée.

Il est fort probable qu'il ne comprenait pas le suédois, car il se trompe sur le contenu du manuscrit : ce dernier n'était pas fait pour l'instruction des Lapons, mais à usage d'enseignement, au sujet des Lapons, pour les suédophones. Ce n'était donc pas la valeur informative 
de l'objet qui l'intéressait, mais sa valeur idéelle et symbolique comme témoin d'un milieu culturel vivant : il indique que l'auteur du manuscrit, Olaus Graan, était musicien de la fameuse reine de Suède, Christine, et membre de la famille d'un écrivain dont s'est inspiré l'auteur reconnu du premier livre moderne sur la Laponie (paru en 1673), Johannes Schefferus. La Lapponia de ce dernier fut un succès immédiat sur le marché des livres de géographie culturelle; il a été rédigé en latin et tout de suite traduit en anglais, français, allemand et néerlandais.

Pour cet inconnu du $18^{\mathrm{e}}$ siècle, la valeur du manuscrit s'explique donc par sa participation symbolique à la

vie et à l'activité de deux acteurs culturels et politiques auxquels il attache une grande importance - la reine Christine et Johannes Schefferus. Ce fut donc probablement pour lui une bonne raison de le garder dans la collection dont il faisait partie avant d'être intégré à celle de la Kaiserliche Universitäts- und Landesbibliothek de Strasbourg après 1871.

Mis à part Olaus Graan, le premier nom cité par notre commentateur anonyme reste de nos jours largement connu en dehors du cercle des spécialistes. La reine Christine avait pendant un certain temps gouverné la Suède dans une période de puissance pour le pays. Son père Gustave II Adolphe était intervenu avec un grand succès pendant la guerre de Trente Ans, aux côtés des protestants, et son succès militaire avait surpris et intrigué ses contemporains, la Suède étant une " terra incognita ", presque totalement inconnue pour les acteurs d'autres pays européens, et pour cette raison souvent imaginée comme fondamentalement non civilisée. La propagande de ses ennemis proposa beaucoup de raisons pour expliquer ce succès. Une de ces raisons nous intéresse tout particulièrement : on soupçonnait les Suédois d'employer des magiciens lapons.

À la mort de son père en 1632, Christine avait cinq ans. Elle fut cependant reine de Suède jusqu'à sa conversion au catholicisme en 1654. Cette période est caractérisée par l'effort suédois d'ajouter à des compétences militaires désormais reconnues une reconnaissance culturelle sur la scène européenne. Parmi les nombreuses actions entreprises par le gouvernement dans ce but, il convient de citer l'invitation faite aux intellectuels et artistes européens les plus connus de venir à la cour de la reine et dans les universités du pays. Une place-clé dans ce " conseil de sagesse " était réservée à René Descartes. Au grand regret de Christine, il décéda quelques mois après son arrivée dans le pays.

Le deuxième nom cité par le commentateur inconnu du manuscrit faisait partie de ce cercle d'intellectuels appelés à développer les compétences scientifiques des milieux universitaires et académiques suédois et, partant, la renommée du pays dans ce domaine. Johannes Schefferus, philologue reconnu et archéologue avant la lettre, a saisi cet appel qui est devenu le fil conducteur de sa vie professionnelle, et peut être considéré aujourd'hui comme l'un des grands innovateurs du champ de la philologie au sens large en Suède, ou pour le dire avec les mots de Sten Lindroth, comme « le créateur de la philologie classique en Suède ". On pourrait également l'appeler le fondateur des études sames, même si cet effort ne fut qu'un produit secondaire de ses activités. Rien ne semblait le prédisposer à ce destin à sa naissance. Johannes Schefferus (1621-1680) était originaire de Strasbourg. Né dans une famille aisée, Schefferus fit sa scolarité et une grande partie de ses études universitaires dans sa ville natale. Il y étudia particulièrement la philologie avec Matthias Bernegger, Johannes Henricus Boeclerus et Johan Freinshemius, trois grands noms de la philologie de son temps. Pour compléter son éducation, il fit des voyages d'études en Suisse et dans les ProvincesUnies néerlandaises, surtout à l'université de Leyde, où il suivit les cours de Gerhard Johan Vossius, Marcus Zueris Boxhornius, Daniel Heinsius, Petrus Cunæus, Hugo Grotius et beaucoup d'autres enseignant dans ce grand centre intellectuel européen.

L'intérêt principal des philologues strasbourgeois, dont il faisait partie, était l'édition et l'étude des écrivains classiques et donc latins. On peut citer ainsi son édition de la Varia historia de Claudius Aelianus, imprimée à Strasbourg en 1647. Une spécificité strasbourgeoise était cependant la curiosité particulière pour la culture matérielle des civilisations de l'Antiquité. Dans cet esprit, Schefferus rédigea l'une de ses premières publications sur les différents types de bateaux connus par les Anciens, la Dissertatio de varietate 
navium, imprimée à Strasbourg en 1643 - champ d'investigation qu'il continuera à travailler en Suède avec des publications comme De militia navali veterum libri quatuor. Ad historiam grcecam latinamque utiles, publiée à Upsal en 1654, ou encore De re vehiculari veterum, publiée à Francfort en 1671. La philologie n'était donc pas pour lui uniquement une question de mots, mais aussi de choses. C'était une philologie du réel, basée sur l'étude des textes.

C'est avec ce bagage, et sur la recommandation de son professeur Johann Henrik Boeclerus, qu'il fut invité en Suède pour devenir professeur d'éloquence et de politique à Skytte, l'une des chaires universitaires suédoises les plus reconnues, à l'âge de vingt-sept ans. Boeclerus le rejoignit plus tard en Suède comme historien du royaume (Rikshistoriograf) de 1649 à 1652. Son prédécesseur était Johan Freinshemius, beau-fils de l'autre professeur de Scheffer, Bernegger, également représentant de la philologie strasbourgeoise de l'époque.

Entre Strasbourg et la cour de Suède existaient donc des échanges intellectuels et personnels significatifs. Cependant, ces rapports " bilatéraux» faisaient partie d'un réseau professionnel plus important, qui devient visible quand on étudie les provenances des autres collègues étrangers de Schefferus en Suède. Parmi eux, il convient de citer Nicolaus Heinsius, Claudius Salmasius ou Isaac Vossius, tous des savants issus du milieu académique de Leyde. L'intérêt porté par les Suédois aux intellectuels de Strasbourg, et les relations qui s'ensuivirent, étaient vraisemblablement facilités par un réseau rhénan préétabli. Ils ont été employés en tant que membres de ce réseau : selon Allan Ellenius par exemple, la méthode philologique néerlandaise - ou devrait-on l'appeler plutôt " rhénane " ? - était considérée comme à la pointe de ce que produisait le monde d'alors.

Parmi tous ses collègues provenant d'autres pays, Schefferus fut peut-être celui qui s'investit le plus dans la politique et les intérêts de son employeur, sans pour autant y perdre son autonomie scientifique. Pour introduire les méthodes philologiques de la région rhénane en Suède en particulier et dans le monde savant en général, il rédigea des manuels et établit des éditions d'auteurs classiques pour l'enseignement. Plus importante, cependant, fut sa participation à la grande aventure historiographique suédoise, le gothicisme - implication qui toutefois n'alla pas sans complications.

Quand la Suède avait accédé à sa position de puissance pendant la guerre de Trente Ans, la question de son histoire était devenue urgente. Bien évidemment, elle ne pouvait pas revendiquer une appartenance à la tradition gréco-romaine autrement que par un apprentissage tardif. Les républiques italiennes, le royaume français, même le Saint-Empire romain dit germanique pouvaient expliquer leur descendance des Anciens de façon relativement convaincante. Pour les Suédois, par contre, ce chemin était difficile. Ils eurent recours pour cela à la théorie de leur origine gothique.

Celle-ci n'était pas nouvelle en soi. Nous savons que l'évêque Nils Ragvaldsson l'avait déjà exprimée lors du concile de Bâle en 1434, et quand les frères Johannes et Olaus Magnus rédigèrent leurs histoires et descriptions des pays nordiques au $16^{\mathrm{e}}$ siècle en Italie, afin de susciter un intérêt pour ces pays abandonnés à la Réforme, ils utilisèrent la descendance supposée des peuples nordiques $\mathrm{du}$ peuple guerrier qui avait conquis l'Empire romain sous la conduite du roi Théodoric. Si l'on n'appartenait pas aux Romains, au moins, on était la cause de la fin de leur période de gloire! De plus, selon l'historiographe des Goths du $6^{e}$ siècle, Jordanès, le Nord et donc probablement la Scandinavie étaient le berceau de tous ces peuples guerriers, formés par son climat hostile ; la région était l'" officina gentium ", ou encore la " vagina nationum ". La Suède n'était-elle donc pas un pays d'une grande importance culturelle, le lieu d'une autre Antiquité ? De cette manière, la " terra incognita " de la Scandinavie devenait une " terra heroica ", et un pays significatif pour l'histoire européenne.

Ces efforts n'eurent pas un grand impact jusqu'au $17^{\mathrm{e}}$ siècle. La situation changea après la guerre de Trente Ans, et Schefferus y fut impliqué. Les familles royales et nobles de la Suède investirent beaucoup de moyens pour aboutir à la reconstruction et à la visibilité d'un passé supposé glorieux. Des acheteurs de manuscrits étaient envoyés en Islande pour y chercher des sources écrites ; elles étaient par la suite éditées, traduites et analysées. Les pierres runiques et autres sources du patrimoine historique étaient inventoriées, décrites et interprétées, les premières fouilles archéologiques conduites de façon méthodique... Toute la gloire historique que l'on en attendait devait ensuite rayonner sur la Suède.

Pour organiser ces efforts fut fondé en 1667 une sorte de " think tank " historique, le Collegium Antiquitatum à Upsal. Parmi ses membres figuraient Georg Stiernhielm, Johannes Loccenius, Olof Verelius, Johan Hadorph, et bien entendu Johannes Schefferus. Les travaux de ce collectif érudit étaient suivis par les savants étrangers, particulièrement dans les pays germanophones, qui étaient engagés dans des projets similaires, mais moins bien financés. 


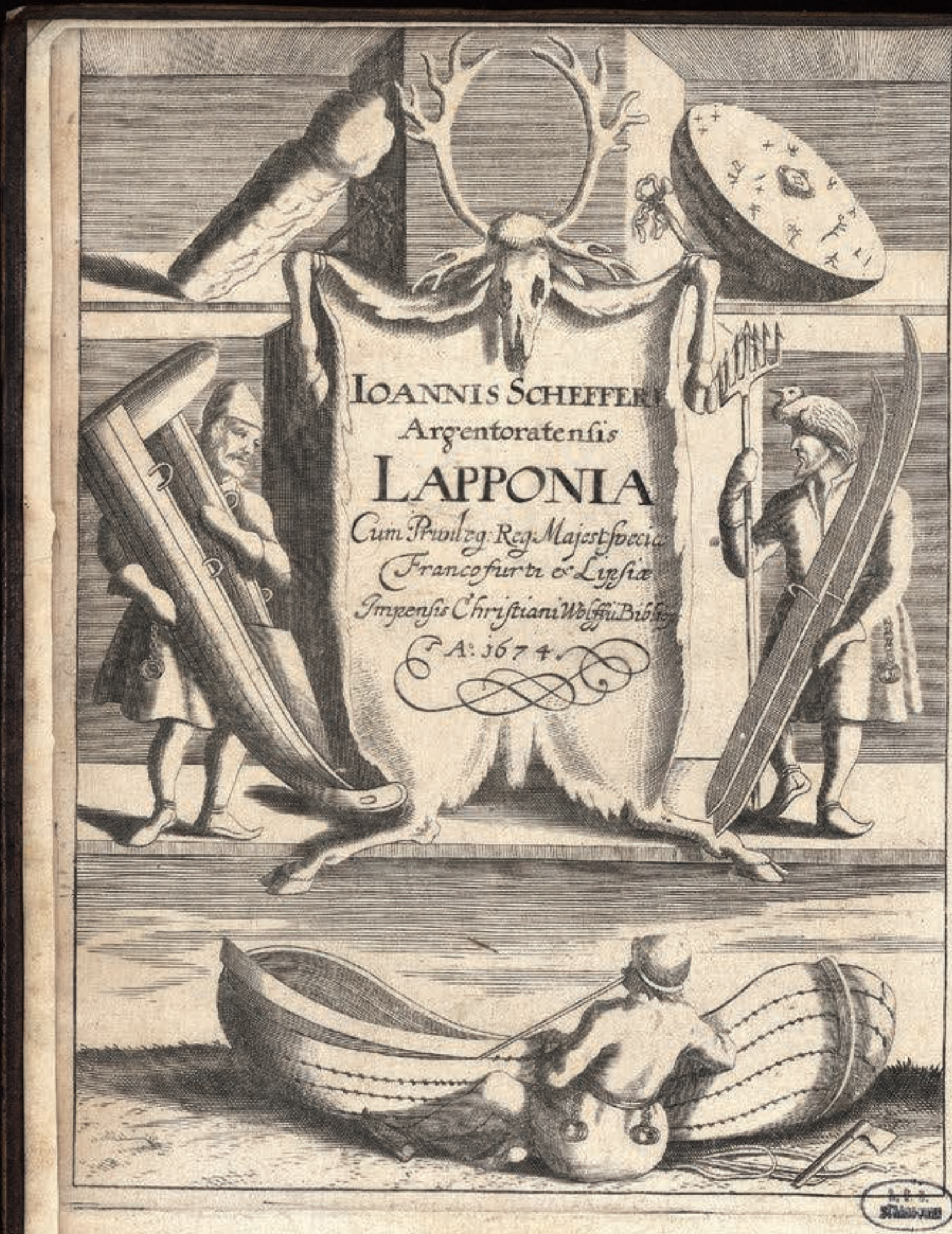


R103026

Gcorgy Stokari $d 68 j$.

\section{IOANNIS SCHEFFERI}
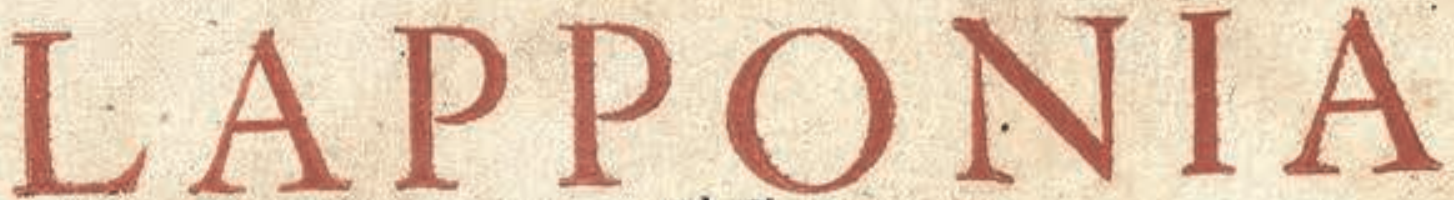

Id est,

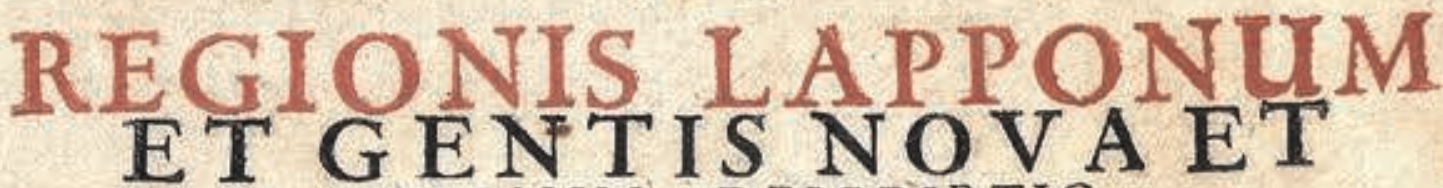

VERISSIMA DESCRIP TIO.

In qua multa

De origine, fuperftitione, facris magicis, victu,cultu,negotiis Lapponum, item Animalium, metallorumque indole, qux in terris eorum proveniunt,

hactenus incognita

Produntur, breiconibus adjectis cum cura illuflrantsr.

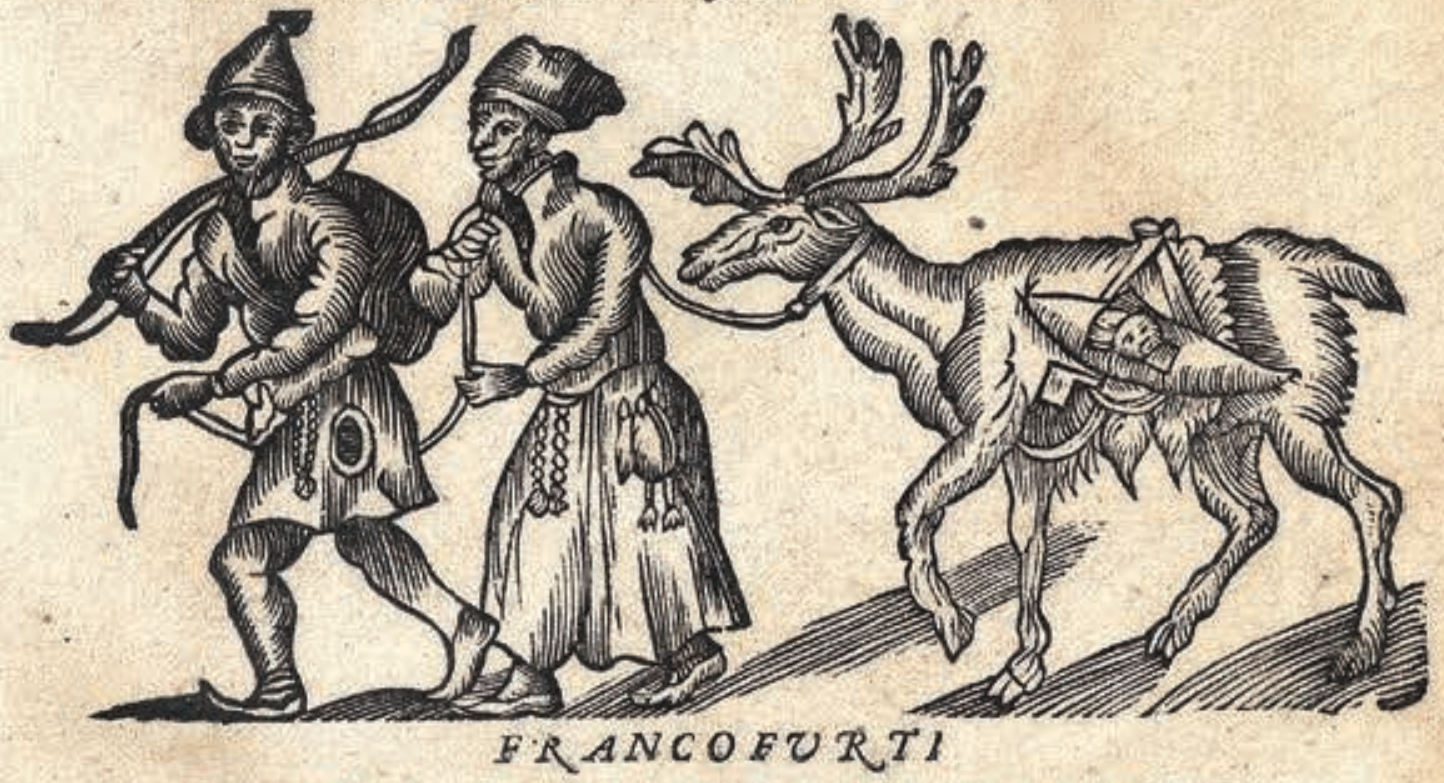

Es Officina CHRISTIANI WOLFFII

Typis TOANNIS ANDREA.

ANDO M. DC. IXXIII. 
L'ouvrage le plus conséquent et le plus connu de cette école, par contre, est l'œuvre d'Olof Rudbeck, savant extérieur à ce groupe, mais proche de son esprit et lié à lui par des rapports personnels. Dans son Atland eller Manheim Atlantica sive Manheim, vera Japheti posterum sedes ac patria, publié à partir de 1679, Rudbeck entreprend un vaste travail d'histoire et de philologie comparatives pour montrer que la Suède était identique à l'Atlantide de Platon et, par conséquent, elle aussi un berceau de l'humanité. Aujourd'hui, les méthodes employées par Rudbeck ne sont plus considérées comme scientifiques, et le résultat auquel il arrive est par là peu crédible. À la fin du $17^{\mathrm{e}}$ siècle, il en allait autrement. Rudbeck avait beaucoup d'admirateurs, même en dehors de la Suède, et les critiques formulées à son encontre étaient souvent plus le fait d'un patriotisme différent du sien que d'une critique de sa méthode ${ }^{2}$. Il est évident que le patriotisme, la volonté de mettre la Suède et ses familles royales et nobles en avant, dans un contexte de montée en puissance, étaient à la base des efforts des érudits suédois, et que cet amour pour la patrie facilitait parfois l'acceptation de reconstructions historiques très spéculatives et les inconséquences méthodologiques que celles-ci pouvaient occasionner. Allan Ellenius souligne toutefois que cet élan patriotique était moins virulent chez Schefferus, peut-être en raison de son origine, mais surtout à cause de sa formation universitaire philologique qui le distinguait de ses collègues. Ses propres travaux dans ce contexte témoignent par conséquent d'une distance scientifique par rapport aux rêves gothiques et aux controverses que ces derniers pouvaient occasionner - comme ils témoignent aussi des innovations philologiques et archéologiques du temps.

Pour Schefferus en effet, la méthode scientifique revendiquait d'abord l'établissement d'un inventaire de toutes les sources disponibles sur un sujet donné, ainsi que l'examen critique des vestiges archéologiques et des autres témoins matériels. Ces analyses le conduisirent entre autres à la contestation de la fiabilité des traditions orales, que ses collègues avaient utilisées pour remonter au temps d'Abraham, comme en témoigne la datation, finalement du $15^{\mathrm{e}}$ siècle, d'une statue qui auparavant était considérée comme un reste de l'ancien temple païen d'Upsal. Le grand chancelier du royaume, Magnus De la Gardie, et d'autres considéraient ses travaux d'ailleurs avec inquiétude pas forcément en raison des idées de Schefferus, mais à cause des conséquences de celles-ci pour la réputation des études menées en Suède sur le passé du pays. Car si Schefferus avait raison, cela signifiait que les travaux des autres grands noms suédois étaient devenus obsolètes.
Par une ironie de l'Histoire, le nom de cet esprit critique et indépendant sera surtout associé pour la postérité à un sujet et à une région dont il ne savait presque rien et qu'il n'a jamais lui-même visitée : la Laponie. La Lapponia, publiée en 1673 à Francfort et tout de suite traduite en plusieurs langues (entre autres en français, en 1678, sous le titre Histoire de la Laponie : sa description, l'origine, les mœurs, la manière de vivre de ses habitans, leur religion, leur magie, $\checkmark$ les choses rares du païs), était une commande du grand chancelier du royaume déjà cité, Magnus De la Gardie. Dans sa préface, qui ne fut malheureusement pas traduite en français, il explique les raisons qui ont conduit à ce travail : De la Gardie avait vu que « circumferri pluscula de Lapponibus apud exteros, quædam fabulis anilibus, quam veritati propiora ; quædam etiam in odium gentis Sueticæ, \& diminutionem gloriæ, quam armis victricibus per tot annos sibi peperit, labemque conficta ; interesse Patriæ putavit, ne sineret diutius in tenebris latere, quæ ad mores hujus gentis, indolemque, ad ingenium regionis, in qua degit rectius noscenda possunt pertinere $" 3$. Son but était donc explicitement politique : il fallait montrer la vraie nature des Lapons, car les rumeurs qui circulaient sur eux en Europe étaient problématiques pour la réputation de la Suède.

Cependant, comment procéder si l'on n'a pas de connaissances, à l'instar du professeur de rhétorique et d'histoire qu'était Schefferus - et ce d'autant plus que la Laponie est loin d'Upsal ? Il est intéressant de noter qu'il se met en scène dans un mouvement d'une « double étrangeté ". D’après les indications de la couverture, il est originaire de Strasbourg (Argentoratensis), et non d'Upsal. D'une certaine manière, il apparaît de cette façon plus objectif et plus proche de son lecteur potentiel. La préface le souligne : ily dit qu'il habite un lieu où il est aussi étranger que les Lapons. Upsal devient de cette façon un lieu tiers, un endroit de rencontre qui n'est ni son propre habitat naturel ni celui de son objet d'études, les Lapons un lieu neutre qui aide à l'objectivité de son propos.

Cet inconvénient se transforme tout de suite en avantage : il peut utiliser sa méthode, formée aux sources classiques, à la description de la Laponie. D'abord, il fait un inventaire de toutes les sources écrites qu'il peut trouver, c'est-à-dire des sources anciennes, celles du moyen-âge scandinave, mais aussi des rapports et des relations des prêtres qui travaillent en Laponie. Ces rapports avaient été demandés par De la Gardie auparavant. De plus, il rencontre des étudiants venant de la région, avec lesquels il ne mène apparemment pas des entretiens à proprement parler, mais à qui il demande de rédiger des textes. Ces derniers vont être utilisés de la même façon que les autres textes écrits. 

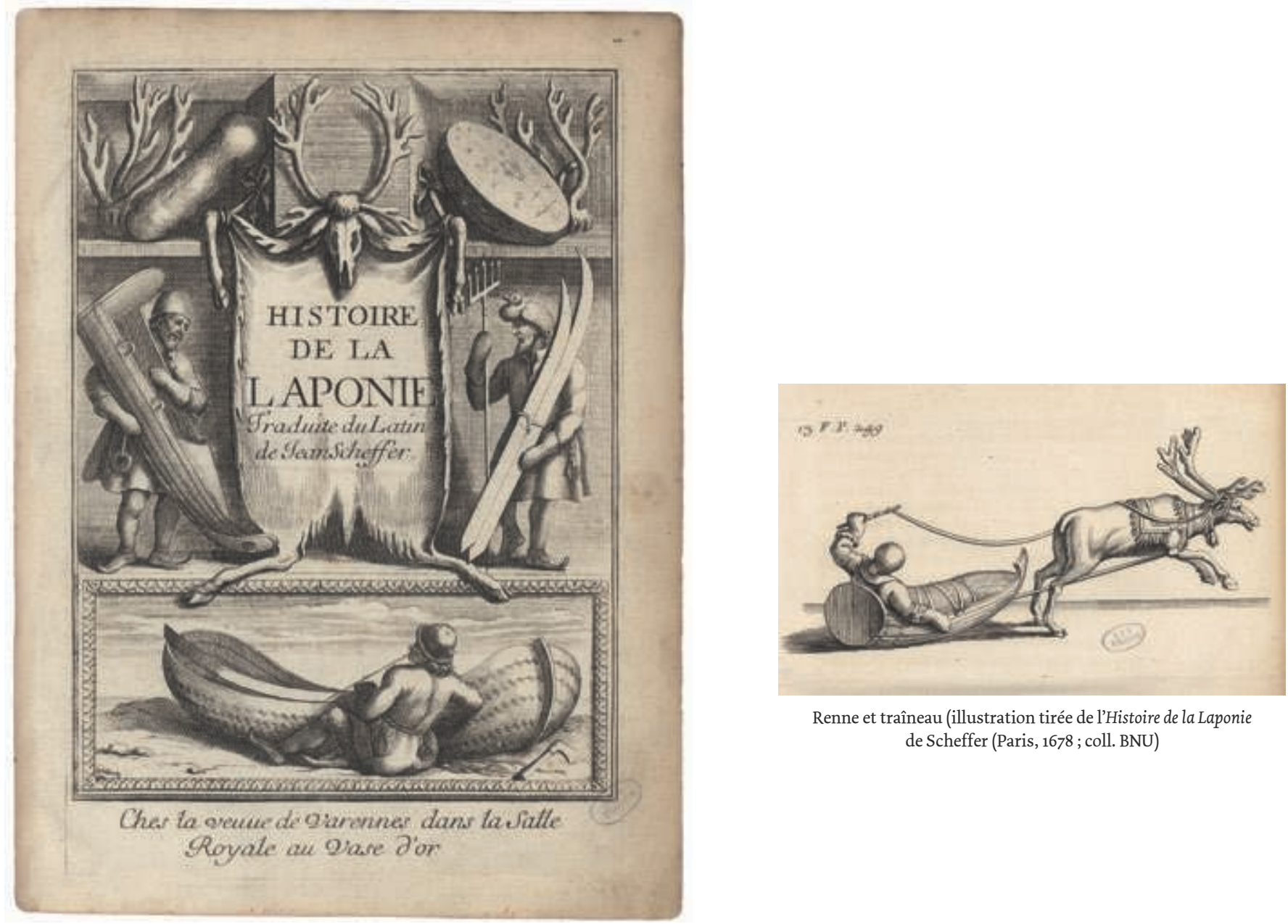

Renne et traîneau (illustration tirée de l'Histoire de la Laponie de Scheffer (Paris, 1678 ; coll. BNU)

Avant-titre de l'édition française de l'Histoire de la Laponie de Scheffer (Paris, 1678 ; coll. BNU)

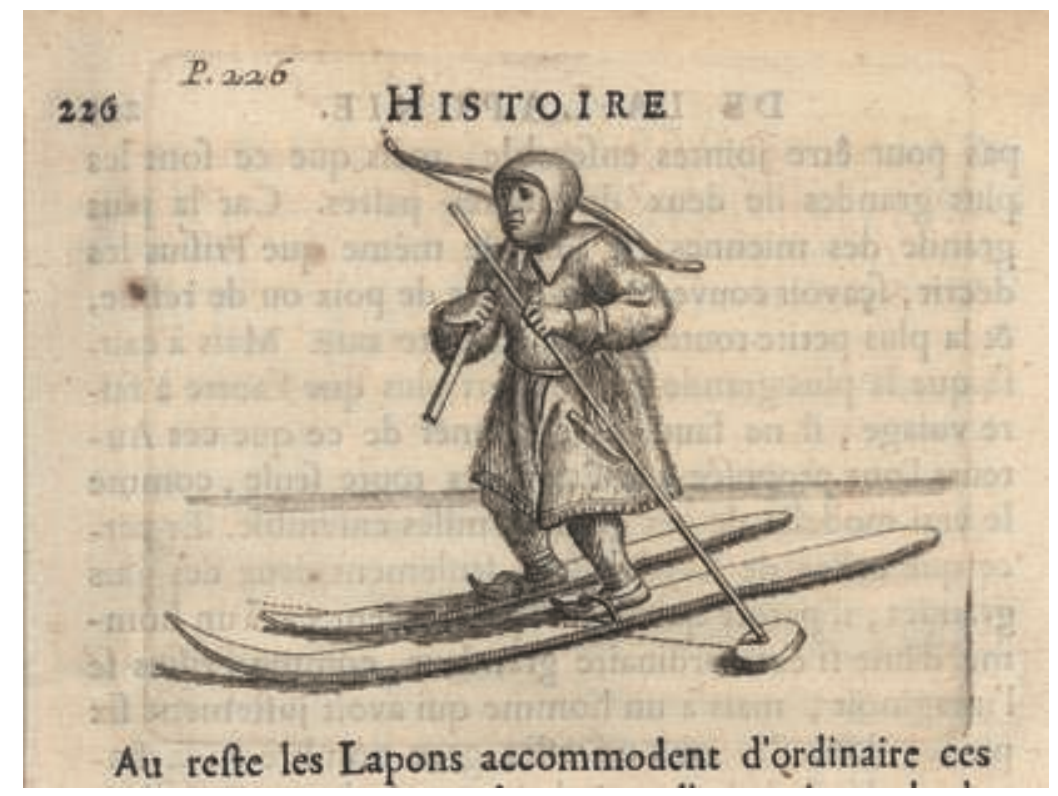

Un homme à skis (illustration tirée de l'Histoire de la Laponie de Scheffer, Paris, 1678 ; coll. BNU) 
Intéressante aussi est la table des auteurs qu'il utilise. Nous y trouvons les savants scandinaves du " gothicisme" d'un côté, leurs prédécesseurs comme les frères Johannes et Olaus Magnus, Arngrimr Jonsson, Ole Worm, Johannes Bazius, Andreas Buræus, Adam de Brême, Saxo Grammaticus, mais aussi des érudits rhénans : Philipp Cluverius, Hugo Grotius, Sebastian Münster et Albertus Magnus. Son étude est donc une analyse philologique, basée sur des textes. Cependant, il fouilla aussi les archives royales et particulièrement les cabinets de curiosités, et en premier lieu celui de De la Gardie, pour intégrer la culture matérielle à sa réflexion. On a donc affaire, dans le cas de la Laponie aussi, à une "philologie des mots et des choses".

La structure de l'ouvrage témoigne d'un esprit méthodologique et philologique. Le premier chapitre traite du " nom de la Laponie, \& de celuy de ses Peuples "; s'ensuivent des rapports sur " la situation de la Laponie ", " du climat, \& et de la nature du Païs », la " division de la Laponie ", " de la manière dont sont faits les Lapons, leur naturel, \& du caractère de leur esprit ", " de l'origine des Lapons ", " de la première religion des Lapons ", " de la seconde religion des Lapons qui est la chrétienne ", etc. au total 35 chapitres. Cette forme tout à fait classique et inspirée par l'esprit de méthode sert vraisemblablement à diminuer l'exotisme du pays, et un chapitre surprenant comme celui sur « la seconde religion des Lapons" ne fait qu'abonder dans ce sens, en soulignant l'état civilisé de ces peuples.

Les chapitres eux-mêmes ressemblent souvent à des collections de citations de toutes les sources de Schefferus, dont il fait parfois à la fin une synthèse critique. Le discours devient de cette manière polyphonique; la Laponie est dans son livre un paysage composé de voix différentes qui se soutiennent l'une l'autre. L'effet recherché est celui de l'objectivité de l'auteur, qui n'invente rien lui-même, mais qui rassemble et compare avec esprit critique les différentes perspectives.

$\mathrm{Au}$ cours de ses analyses, Schefferus traite aussi de l'appartenance linguistique du peuple étudié. Il souligne, tout à fait correctement, que les langues sames n'ont rien à voir ni avec le suédois, ni avec le russe, mais qu'elles sont plutôt à rapprocher des langues finnoise et samoyède. On pourrait presque voir en lui l'un des fondateurs de la linguistique des langues finno-ougriennes. Dans son analyse du caractère du peuple, les Sames apparaissent comme différents des Suédois ; Schefferus souligne qu'ils ne sont pas très doués pour la guerre - à la différence des Suédois - mais qu'ils sont des êtres humains tout à fait capables d'être civilisés, et donc à considérer à l'instar des autres peuples.
Dans ce paysage textuel, un certain nombre de textes ressortent - en particulier deux chants sames qu'il cite en langue originale pour les traduire ensuite en latin. L'effet recherché est celui de l'authenticité, un " effet du réel ", pour parler comme Roland Barthes. En s'appuyant sur ses informateurs sames, Schefferus raconte des scènes de marché, en hiver et en été, où de jeunes Sames utilisent des chants pour déclarer leur amour, il les cite - et ce faisant séduit son lecteur qui devient témoin de la vie du peuple.

« Kulnasatz, mon petit Renne, il faut nous hâter, car nous avons du chemin à faire, les terres humides sont vastes. Tu ne me seras pas toutefois ennuyeux marais Kaige, marais Kailvva je te dis à dieu. Plusieurs pensées roulent dans mon esprit, lorsque je suis porté par le marais Kaige. Mon Renne nous sommes agiles \& légers, ainsi nous verrons plûtost la fin de nôtre travail, \& nous arriverons où nous avons résolu d'aller; je verrai là ma maîtresse aller à la promenade. Kulnasatz, mon Renne regarde \& considère, si tu n'aperceveras point qu'elle se lave ${ }^{4}$.

Un lecteur lointain, mais qui deviendra fameux, Johann Gottlieb Herder, se laissera séduire à un siècle de distance, et sera amené à utiliser le matériau laissé par Schefferus comme point de départ de sa réflexion sur la chanson populaire et de son anthologie Stimmen der Völker in Liedern, dont on sait l'influence qu'elle a eue par la suite.

Le succès de son livre fut donc peut-être dû à la double étrangeté que Schefferus mit en scène. La Laponie, alors inconnue, était décrite dans un langage connu du public cultivé européen : c'était celui de la philologie classique, telle qu'elle était pratiquée dans les milieux rhénans, enrichie par l'étude, conçue dans le même esprit, des antiquités et de la culture matérielle. L'exotisme du Nord était domestiqué, compréhensible, détaché de son contexte suédois par la forme, la méthode et même par une idéologie qui s'abstenait de références trop appuyées au " gothicisme ".

Le rapport manuscrit d'Olaus Graan qui se trouve à Strasbourg n'a pas servi à Schefferus ; il fut réalisé vingt ans après la parution de l'œuvre de ce dernier, et quelques années après sa mort. Il montre, quoi qu'il en soit, que les échanges entre le Grand Nord et la région rhénane étaient bien établis. Les compétences linguistiques, à l'époque, n'étaient peut-être pas toujours suffisamment développées pour qu'un nombre important de lecteurs en profite. Aujourd'hui, sa présence à Strasbourg montre que l'intérêt pour cette immense partie de l'Europe, et la conscience de son importance pour notre histoire commune, sont toujours d'actualité.

\section{Thomas Mohnike}




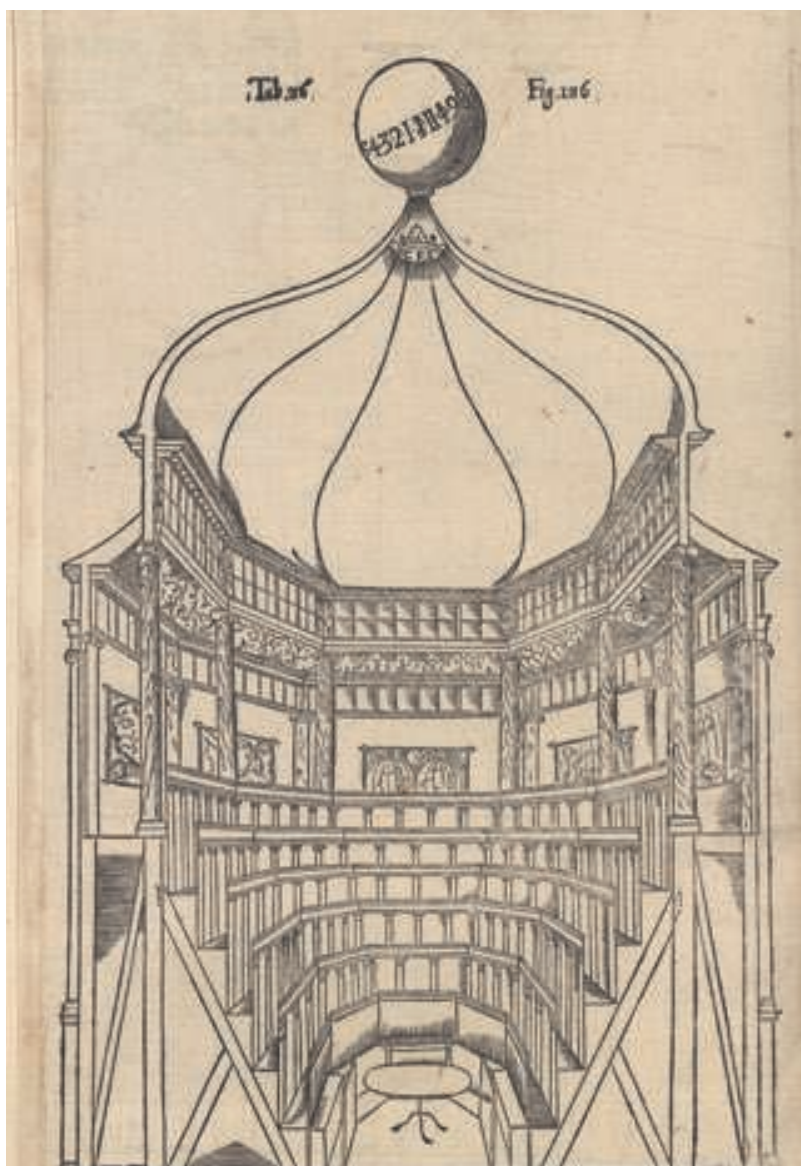

Planche extraite de l'atlas complétant l'Atlantica sive Manheim de Rudbeck (Uppsala, 1679) et représentant le théâtre anatomique d'Uppsala, construit sous la direction de Rudbeck (coll. BNU)

\section{ORIENTATIONS BIBLIOGRAPHIQUES :}

Sonia Brough / The Goths and the concept of Gothic in Germany from 1500 to 1750, Bern, Peter Lang, 1985

Allan Ellenius / Johannes Schefferus and Swedish Antiquity, in Journal of the Warburg and Courtauld Institutes, 20 (1957), $\mathrm{n}^{\circ} 1 / 2, \mathrm{p} \cdot 59-74$

Christian Jacob (dir.) / Lieux de savoir, Paris, Albin Michel, 2007

Sten Lindroth / Svensk Lärdomshistoria, Stormaktstiden, Stockholm, Norstedts förlag, 1975

David N. Livingstone / Putting science in its place : geographies of scientific knowledge, Chicago, University of Chicago Press, 2003

Erland Sellberg / Johannes Schefferus och hans roll, in Centralantikvariatet, Johannes Schefferus och fyra andra utländska lärda i Stormaktstidens Sverige, Stockholm, Centralantikvariatet, 2008, p. 7-16

Stig Strömholm / Johannes Schefferus, un Strasbourgeois en Suède, in Société savante d'Alsace et des régions de l'Est (éd.), L'Europe, l'Alsace et la France. Problèmes intérieurs et relations internationales à l'époque moderne. Etudes réunies en l'honneur du doyen Georges Livet pour son $70^{\circ}$ anniversaire, Colmar, Editions d'Alsace, 1986, p. 302-306

4 - In Johannes Scheffer, Histoire de la Laponie : sa description, l'origine, les mours, la maniere de vivre de ses habitans, leur religion, leur magie, \& les choses rares du païs. Avec plusieurs additions \& augmentations fort curieuses, qui jusques-icy n'ont pas esté imprimées. Traduites du latin de Monsieur Scheffer. Par L[e] P[ère] A[ugustin] L[ubin] geographe ordinaire de sa Majesté (Paris, Veuve Olivier de Varennes, 1678), p. 263. Nous avons gardé l'orthographe et la graphie du texte original. 